\title{
Stabilization and Analytic Approximate Solutions of an Optimal Control Problem
}

https://doi.org/10.1515/phys-2018-0064

Received Mar 14, 2017; accepted Jun 22, 2018

\begin{abstract}
This paper analyses a dynamical system derived from a left-invariant, drift-free optimal control problem on the Lie group $S O(3) \times \mathbb{R}^{3} \times \mathbb{R}^{3}$ in deep connection with the important role of the Lie groups in tackling the various problems occurring in physics, mathematics, engineering and economic areas [1-5]. The stability results for the initial dynamics were inconclusive for a lot of equilibrium points (see [6]), so a linear control has been considered in order to stabilize the dynamics. The analytic approximate solutions of the resulting nonlinear system are established and a comparison with the numerical results obtained via the fourth-order Runge-Kutta method is achieved.
\end{abstract}

Keywords: ordinary differential equations, solution of equations, optimal control, nonlinear stability, optimal homotopy asymptotic method

PACS: 02.60.Cb, 02.60.Lj

\section{Introduction}

In the last of years, the optimal control problems on the Lie groups has drawn a lot of attention. The opportunity to study a mechanical system using geometric tools have had a big success in many areas of physics (see for instance [7-11]), engineering [12-15] and biology [16]. The left-invariant, drift-free control systems on the Lie groups belong to this class; having a Lie group as space configuration, the existence of a Hamilton-Poisson formulation is assured and therefore several powerful techniques can be

\footnotetext{
Camelia Pop: University Politehnica Timişoara, Department of Mathematics, 300006 Timişoara, Romania;

Email: cariesanu@yahoo.com

Camelia Petrişor: University Politehnica Timişoara, Department of Mathematics, 300006 Timişoara, Romania;

Email: galeacamelia@yahoo.com

*Corresponding Author: Remus-Daniel Ene: University Politehnica Timişoara, Department of Mathematics, 300006 Timisoara, Romania; Email: remus.ene@upt.ro
}

employed such as the reduction of the symmetric system, the determination of the relative equilibria from a global point of view, the energy methods for studying the stability, specific methods for finding the periodical orbits, the variational integrators for greater accuracy in the numerical simulation and the geometric control theory for control problems. Using the Lie group framework of the system, the determination of the equilibria and the energy method for determining the nonlinear stability, were performed in [6]. However, the nonlinear stability of some equilibria has remained unsolved, both methods - the energyCasimir and Arnold's - being unsuccessful.

The Hamilton-Poisson systems play an important role in mathematics, geometrical mechanics and physics (classical, quantum, or relativistic) due to their deep connections to metriplectic systems and, being useful to achieve their syncronization in information security and cryptography [17-20].

In this paper, a linear control function is proposed in order to stabilize an equilibria and the analytic approximate solutions via the Optimal Homotopy Asymptotic Method (OHAM) of the controlled system are presented.

\section{The Geometrical Framework of the Problem and the Stabilization by one Linear Control}

To begin with let us introduce very briefly the leftinvariant, drift-free control system on the Lie group $G=$ $S O(3) \times \mathbb{R}^{3} \times \mathbb{R}^{3}$ considered in [6]

$$
\dot{X}=X\left(A_{1} u_{1}+A_{2} u_{2}+A_{3} u_{3}+A_{5} u_{5}+A_{7} u_{7}\right),
$$

where $X \in G, u_{i}, i=\overline{1,9}$ are the controls and $\left\{A_{i}\right\}_{i=\overline{1,9}}$ is the standard basis of the corresponding Lie algebra $g$. Then, the following results hold:

Proposition 2.1. [6]

- The system (1) is controllable.

- The controls that minimize the function

$$
J\left(u_{1}, u_{2}, u_{3}, u_{5}, u_{7}\right)=
$$




$$
=\frac{1}{2} \int_{0}^{t_{f}}\left[u_{1}^{2}(t)+u_{2}^{2}(t)+u_{3}^{2}(t)+u_{5}^{2}(t)+u_{7}^{2}(t)\right] d t
$$

and steer the system (1) from $X=X_{0}$ at $t=0$ to $X=X_{f}$ at $t=t_{f}$ are given by the solutions of

$$
\begin{cases}\dot{x}_{1}=-x_{5} x_{6}, & \dot{x}_{2}=x_{7} x_{9}, \\ \dot{x}_{3}=x_{4} x_{5}-x_{7} x_{8}, & \dot{x}_{4}=-x_{2} x_{6}+x_{3} x_{5}, \\ \dot{x}_{5}=x_{1} x_{6}-x_{3} x_{4}, & \dot{x}_{6}=-x_{1} x_{5}+x_{2} x_{4}, \\ \dot{x}_{7}=-x_{2} x_{9}+x_{3} x_{8}, & \dot{x}_{8}=x_{1} x_{9}-x_{3} x_{7}, \\ \dot{x}_{9}=-x_{1} x_{8}+x_{2} x_{7} . & \end{cases}
$$

The main goal of this section is to establish some stability results of the equilibrium points $e_{8}^{M N P}=$ $\left(M, 0, N, P, 0, \frac{N P}{M}, 0,0,0\right), M, N, P \in \mathbb{R}^{\star}$, of the above system. Some stability results regarding the equilibrium states

$$
\begin{aligned}
e_{1}^{M N P Q} & =(0,0,0, M, 0, N, 0, P, Q), M, N, P, Q \in \mathbb{R}, \\
e_{2}^{M N P} & =(0,0, M, 0,0, N, 0,0, P), M, N, P \in \mathbb{R}, \\
e_{3}^{M N P} & =(0,0,0,0, M, 0,0, N, P), M, N, P \in \mathbb{R}, \\
e_{4}^{M N P} & =(0, M, 0,0, N, 0,0, P, 0), M, N, P \in \mathbb{R}, \\
e_{5}^{M N P} & =(M, N, P, 0,0,0,0,0,0), M, N, P \in \mathbb{R}
\end{aligned}
$$

were already obtained in [6], but the stability problem for the other equilibrium states remains unsolved.

In order to stabilize the equilibrium states $e_{8}^{M N P}$ of the system (2) we employ the linear control $u \in C^{\infty}\left(\mathbb{R}^{9}, \mathbb{R}\right)$ given by

$$
\begin{aligned}
& u(\bar{x})=\left(-N x_{2}+N x_{5},-M x_{3}+N x_{1}+M x_{6}-N x_{4},\right. \\
& M x_{2}-M x_{5},-N x_{5}, N x_{4}-M x_{6}, M x_{5},-N x_{8}, \\
& \left.-M x_{9}+N x_{7}, M x_{8}\right),
\end{aligned}
$$

so the controlled system (2)-(3) can be explicitly written:

$$
\left\{\begin{array}{l}
\dot{x}_{1}=-x_{5} x_{6}-N x_{2}+N x_{5} \\
\dot{x}_{2}=x_{7} x_{9}-M x_{3}+N x_{1}+M x_{6}-N x_{4} \\
\dot{x}_{3}=x_{4} x_{5}-x_{7} x_{8}+M x_{2}-M x_{5} \\
\dot{x}_{4}=-x_{2} x_{6}+x_{3} x_{5}-N x_{5} \\
\dot{x}_{5}=x_{1} x_{6}-x_{3} x_{4}+N x_{4}-M x_{6} \\
\dot{x}_{6}=-x_{1} x_{5}+x_{2} x_{4}+M x_{5} \\
\dot{x}_{7}=-x_{2} x_{9}+x_{3} x_{8}-N x_{8} \\
\dot{x}_{8}=x_{1} x_{9}-x_{3} x_{7}-M x_{9}+N x_{7} \\
\dot{x}_{9}=-x_{1} x_{8}+x_{2} x_{7}+M x_{8} .
\end{array}\right.
$$

Proposition 2.2. The controlled system (4) has the Hamilton-Poisson realization

$$
\left(G, \Pi_{-}, H\right)
$$

where $\Pi_{-}$is the minus Lie-Poisson structure on the dual $g^{\star}$ of the Lie algebra, given by

$$
\Pi_{-}=\left[\begin{array}{ccccc}
0 & -x_{3} & x_{2} & 0 & -x_{6} \\
x_{3} & 0 & -x_{1} & x_{6} & 0 \\
-x_{2} & x_{1} & 0 & -x_{5} & x_{4} \\
0 & -x_{6} & x_{5} & 0 & 0 \\
x_{6} & 0 & -x_{4} & 0 & 0 \\
-x_{5} & x_{4} & 0 & 0 & 0 \\
0 & -x_{9} & x_{8} & 0 & 0 \\
x_{9} & 0 & -x_{7} & 0 & 0 \\
-x_{8} & x_{7} & 0 & 0 & 0 \\
x_{5} & 0 & -x_{9} & x_{8} \\
-x_{4} & x_{9} & 0 & -x_{7} \\
0 & -x_{8} & x_{7} & 0 \\
0 & 0 & 0 & 0 \\
0 & 0 & 0 & 0 \\
0 & 0 & 0 & 0 \\
0 & 0 & 0 & 0 \\
0 & 0 & 0 & 0 \\
0 & 0 & 0 & 0
\end{array}\right]
$$

and the Hamiltonian function

$H(\bar{x})=\frac{1}{2}\left(x_{1}^{2}+x_{2}^{2}+x_{3}^{2}+x_{5}^{2}+x_{7}^{2}\right)-M x_{1}-N x_{3}+M x_{4}+N x_{6}$.

Proof. Indeed, it is not hard to see that the dynamics (4) can be put into the equivalent form:

$$
\dot{x}=\Pi_{-} \cdot \nabla H,
$$

as required. More details about Poisson structures can be found in [21].

Proposition 2.3. [6] The functions $C_{1}, C_{2}$ and $C_{3}$ given by:

$$
\begin{gathered}
C_{1}(\bar{x})=\frac{1}{2}\left(x_{4}^{2}+x_{5}^{2}+x_{6}^{2}\right), \\
C_{2}(\bar{x})=\frac{1}{2}\left(x_{7}^{2}+x_{8}^{2}+x_{9}^{2}\right), C_{3}(\bar{x})=x_{1} x_{4}+x_{2} x_{5}+x_{3} x_{6}
\end{gathered}
$$

are Casimirs of our configuration.

Let $B$ be the matrix of linear part of our controlled system (4), that is

$$
B=\left[\begin{array}{ccccc}
0 & -N & 0 & 0 & -\chi_{6}+N \\
N & 0 & -M & -N & 0 \\
0 & M & 0 & x_{5} & \chi_{4}-M \\
0 & -\chi_{6} & x_{5} & 0 & x_{3}-N \\
x_{6} & 0 & -\chi_{4} & -\chi_{3}+N & 0 \\
-\chi_{5} & x_{4} & 0 & x_{2} & -\chi_{1}+M \\
0 & -\chi_{9} & x_{8} & 0 & 0 \\
x_{9} & 0 & -x_{7} & 0 & 0 \\
-x_{8} & x_{7} & 0 & 0 & 0
\end{array}\right.
$$




$\left.\begin{array}{cccc}-x_{5} & 0 & 0 & 0 \\ M & x_{9} & 0 & x_{7} \\ 0 & -x_{8} & -x_{7} & 0 \\ -x_{2} & 0 & 0 & 0 \\ x_{1}-M & 0 & 0 & 0 \\ 0 & 0 & 0 & 0 \\ 0 & 0 & x_{3}-N & -x_{2} \\ 0 & -x_{3}+N & 0 & x_{1}-M \\ 0 & x_{2} & -x_{1}+M & 0\end{array}\right]$.

At the equilibrium of interest, its characteristic polynomial has the following expression

$$
\begin{aligned}
P_{A\left(e_{8}^{M N P}\right)}(\lambda) & =-\lambda^{5}\left[\lambda^{4}+\left(1-\frac{P}{M}\right)^{2}\left(M^{2}+N^{2}\right) \lambda^{2}+\right. \\
+ & \left.\frac{P^{2}}{M^{2}}\left(M^{2}+N^{2}\right)^{2}\left(1-\frac{P}{M}\right)\right] .
\end{aligned}
$$

If $M P<0$, we have five zero eigenvalues and four purely imaginary eigenvalues, so we can conclude:

Proposition 2.4. If $M P<0, M, N, P \in \mathbb{R}^{\star}$, then the controlled system (4) may be spectral stabilized about the equilibrium states $e_{8}^{M N P}$

Moreover we can prove:

Proposition 2.5. If $M P<0, M, N, P \in \mathbb{R}^{*}$, then the controlled system (4) may be nonlinear stabilized about the equilibrium states $e_{8}^{M N P}$.

Proof. Let us consider the function (see [22] for details):

$$
\begin{aligned}
F_{\lambda, \mu}(\bar{x}) & =C_{2}(\bar{x})+\lambda H(\bar{x})+\mu C_{1}(\bar{x}) \\
& =\frac{1}{2}\left(x_{7}^{2}+x_{8}^{2}+x_{9}^{2}\right)+\frac{\lambda}{2}\left(x_{1}^{2}+x_{2}^{2}+x_{3}^{2}+x_{5}^{2}+x_{7}^{2}\right. \\
& \left.-2 M x_{1}-2 N x_{3}+2 M x_{4}+2 N x_{6}\right)+\frac{\mu}{2}\left(x_{4}^{2}+x_{5}^{2}+x_{6}^{2}\right) .
\end{aligned}
$$

Then we have successively:

(i) $\nabla F_{\lambda, \mu}\left(e_{8}^{M N P}\right)=0$ iff $\mu=-\frac{M}{P} \lambda$;

(ii) Considering now

$$
\begin{gathered}
W=\operatorname{ker}\left[d H\left(e_{8}^{M N P}\right)\right] \cap \operatorname{ker}\left[d C_{1}\left(e_{8}^{M N P}\right)\right] \\
=\operatorname{Span}\left\{\left[\begin{array}{l}
1 \\
0 \\
0 \\
0 \\
0 \\
0 \\
0 \\
0 \\
0
\end{array}\right],\left[\begin{array}{l}
0 \\
1 \\
0 \\
0 \\
0 \\
0 \\
0 \\
0 \\
0
\end{array}\right],\left[\begin{array}{l}
0 \\
0 \\
1 \\
0 \\
0 \\
0 \\
0 \\
0 \\
0 \\
0
\end{array}\right],\left[\begin{array}{l}
0 \\
0 \\
0 \\
0 \\
1 \\
0 \\
0 \\
0 \\
0
\end{array}\right],\right.
\end{gathered}
$$

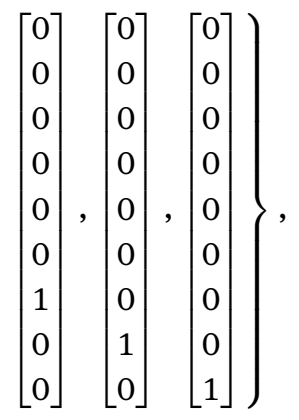

then, for all $v \in W$, i.e. $v=(a, b, c, 0, d, 0, e, f, g)$, $a, b, c, d, e, f, g \in \mathbb{R}$, we have

$$
\begin{aligned}
& v \cdot \nabla^{2} F_{\lambda,-\frac{M}{P} \lambda}\left(e_{8}^{M N P}\right) \cdot v^{t}=\lambda a^{2}+\lambda b^{2}+\lambda c^{2} \\
& +\left(\lambda-\frac{M}{P} \lambda\right) d^{2}+(1+\lambda) e^{2}+f^{2}+g^{2}
\end{aligned}
$$

which is positive definite under the restriction $\lambda>0$ and $M P<0$, and so

$$
\left.\nabla^{2} F_{\lambda,-\frac{M}{P} \lambda}\left(e_{8}^{M N P}\right)\right|_{W \times W}
$$

is positive definite.

Therefore, if $M P<0$, then the equilibrium states $e_{8}^{M N P}$ are nonlinear stable, as required.

\section{Basic Ideas of the Optimal Homotopy Asymptotic Method}

In order to find the approximate analytic solutions of the controlled system (2.4), we shall start with a brief description of this method. For an equation of the form:

$$
\mathcal{L}(x(t))+\mathcal{N}(x(t))=0,
$$

subject to the initial conditions of the type:

$$
x(0)=A, \quad A \in \mathbb{R} \text { - given real number, }
$$

where $\mathcal{L}$ is a linear operator, $\mathcal{N}$ is a nonlinear one and $x(t)$ is the unknown smooth function of the Eq. (5), the approximate analytic solutions can be obtained using the third modified version of Optimal Homotopy Asymptotic Method described hereinafter (see [23] for details).

Following $[23,24]$ we construct the homotopy given by:

$$
\begin{aligned}
& \mathcal{H}\left[\mathcal{L}(X(t, p)), H\left(t, C_{j}\right), \mathcal{N}(X(t, p))\right] \\
& \equiv \mathcal{L}\left(x_{0}(t)\right)++p\left[\mathcal{L}\left(x_{1}\left(t, C_{j}\right)\right)-H\left(t, C_{j}\right) \mathcal{N}\left(x_{0}(t)\right)\right]=0,
\end{aligned}
$$


where $p \in[0,1]$ is the embedding parameter, $H\left(t, C_{j}\right)$, $(H \neq 0)$ is an auxiliary convergence-control function, depending on the variable $t$ and on the parameters $C_{1}, C_{2}$, $\ldots, C_{s}$ and the function $X(t, p)$ has the expression:

$$
X(t, p)=x_{0}(t)+p x_{1}\left(t, C_{j}\right) .
$$

The following properties hold:

$$
\begin{aligned}
& \mathcal{H}\left[\mathcal{L}(X(t, 0)), H\left(t, C_{j}\right), \mathcal{N}(X(t, 0))\right] \\
& \equiv \mathcal{L}\left(x_{0}(t)\right)=0
\end{aligned}
$$

and

$$
\begin{aligned}
& \mathcal{H}\left[\mathcal{L}(X(t, 1)), H\left(t, C_{j}\right), \mathcal{N}(X(t, 1))\right] \\
& \equiv \mathcal{L}\left(x_{1}\left(t, C_{j}\right)\right)-H\left(t, C_{j}\right) \mathcal{N}\left(x_{0}(t)\right)=0 .
\end{aligned}
$$

The governing equations of $x_{0}(t)$ and $x_{1}\left(t, C_{j}\right)$ can be obtained by equating the coefficients of $p^{0}$ and $p^{1}$, respectively:

$$
\begin{gathered}
\mathcal{L}\left(x_{0}(t)\right)=0, \quad x(0)=A, \\
\mathcal{L}\left(x_{1}\left(t, C_{j}\right)\right)=H\left(t, C_{j}\right) \mathcal{N}\left(x_{0}(t)\right), \\
x_{1}\left(0, C_{j}\right)=0, j=\overline{1, s} .
\end{gathered}
$$

The expression of $x_{0}(t)$ can be found by solving the linear Eq. (11). Also, to compute $x_{1}\left(t, C_{j}\right)$ we solve the Eq. (12), by taking into consideration that the nonlinear operator $N$ presents the general form:

$$
N\left(x_{0}(t)\right)=\sum_{k=1}^{m} h_{k}(t) g_{k}(t)
$$

where $m$ is a positive integer and $h_{k}(t)$ and $g_{k}(t)$ are known functions depending both on $x_{0}(t)$ and $N$.

Although the Eq. (12) is a nonhomogeneous linear one in the most cases its solution can not be found.

In order to compute the function $x_{1}\left(t, C_{j}\right)$, we do not solve Eq. (12) but from the theory of differential equation, taking into consideration the method of variation of parameters, the method of influence function, Cauchy method, the operator method [25], it is more convenient to consider the unknown function $x_{1}\left(t, C_{j}\right)$ using the following steps:

- First we consider one of the following expressions for $x_{1}\left(t, C_{j}\right)$

$$
x_{1}\left(t, C_{j}\right)=\sum_{k=1}^{m} H_{k}\left(t, h_{j}(t), C_{j}\right) g_{k}(t), \quad j=\overline{1, s}
$$

or

$$
\begin{aligned}
& x_{1}\left(t, C_{j}\right)=\sum_{k=1}^{m} H_{k}\left(y, g_{j}(t), C_{j}\right) h_{k}(t), \\
& x_{1}\left(0, C_{j}\right)=0, \quad j=\overline{1, s}
\end{aligned}
$$

These expressions of $H_{k}\left(t, h_{j}(t), C_{j}\right)$ contain both linear combinations of the functions $h_{j}$ and the parameters $C_{j}, j=\overline{1, s}$. The summation limit $m$ is an arbitrary positive integer number.

- Next, by taking into account the Eq. (8), for $p=1$, the first-order analytical approximate solution of the Eqs. (5) - (6) is:

$$
\bar{x}\left(t, C_{j}\right)=X(t, 1)=x_{0}(t)+x_{1}\left(t, C_{j}\right) .
$$

- Finally, the convergence-control parameters $C_{1}, C_{2}$, ..., $C_{s}$, which determine the first-order approximate solution (16), can be optimally computed by means of various methods, such as: the least square method, the Galerkin method, the collocation method, the Kantorowich method or the weighted residual method.

In what follows, we will show that the approximate analytic solution $\bar{x}\left(t, C_{j}\right)$ given by Eq. (16) is a weak $\epsilon$ approximate solution of the problem Eq. (5) with the initial condition Eq. (6).

Definition 3.1. [26] We call an $\epsilon$-approximate solution of the problem (5) on the domain $(0, \infty)$ a smooth function $\bar{x}\left(t, C_{j}\right)$ of the form (16) which satisfies the following condition:

$$
\left|R\left(t, \bar{x}\left(t, C_{j}\right)\right)\right|<\epsilon,
$$

together with the initial condition from Eq. (6), where the residual function $R\left(t, \bar{x}\left(t, C_{j}\right)\right)$ is obtained by substituting the Eq. (16) into Eq. (5), i.e.

$$
R\left(t, \bar{x}\left(t, C_{j}\right)\right)=\mathcal{L}\left(\bar{x}\left(t, C_{j}\right)\right)+\mathcal{N}\left(\bar{x}\left(t, C_{j}\right)\right) .
$$

Definition 3.2. [26] We call an weak e-approximate solution of the problem (5) on the domain $(0, \infty)$ a smooth function $\bar{x}\left(t, C_{j}\right)$ of the form (16) which satisfies the following condition:

$$
\int_{0}^{\infty} R^{2}\left(t, \bar{x}\left(t, C_{j}\right)\right) d \eta<\epsilon,
$$

together with the initial condition from Eq. (6). 


\section{Analytic Approximate Solutions of the System (4) using Optimal Homotopy Asymptotic Method}

We now apply the procedure described above to the nonlinear differential system given by the Eqs. (4) with the initial conditions

$$
\begin{aligned}
x_{i}(0) & =A_{i},, \quad A_{i} \in \mathbb{R}-\text { given real number, } \\
i & =\overline{1,9} .
\end{aligned}
$$

Considering the linear operators $\mathcal{L}\left[x_{i}(t)\right]$ :

$$
\begin{aligned}
& \mathcal{L}\left[x_{1}(t)\right]=\dot{x}_{1}(t)+K_{1} x_{1}(t)+N x_{2}(t)-N x_{5}(t), \\
& \mathcal{L}\left[x_{2}(t)\right]=\dot{x}_{2}(t)+K_{1} x_{2}(t)+M x_{3}(t)-N x_{1}(t), \\
& \mathcal{L}\left[x_{3}(t)\right]=\dot{x}_{3}(t)+K_{1} x_{3}(t)-M x_{2}(t)+M x_{5}(t), \\
& \mathcal{L}\left[x_{4}(t)\right]=\dot{x}_{4}(t)+K_{1} x_{4}(t)+K x_{7}(t), \\
& \mathcal{L}\left[x_{5}(t)\right]=\dot{x}_{5}(t)+K_{1} x_{5}(t)+M x_{6}(t), \\
& \mathcal{L}\left[x_{6}(t)\right]=\dot{x}_{6}(t)+K_{1} x_{6}(t)-M x_{5}(t), \\
& \mathcal{L}\left[x_{7}(t)\right]=\dot{x}_{7}(t)+K_{1} x_{7}(t)-K x_{4}(t)+N x_{8}(t), \\
& \mathcal{L}\left[x_{8}(t)\right]=\dot{x}_{8}(t)+K_{1} x_{8}(t)+M x_{9}(t), \\
& \mathcal{L}\left[x_{9}(t)\right]=\dot{x}_{9}(t)+K_{1} x_{9}(t)-M x_{8}(t)
\end{aligned}
$$

and $\mathcal{N}\left[x_{i}(t)\right], i=\overline{1,9}$ :

$$
\begin{aligned}
\mathcal{N}\left[x_{1}(t)\right] & =-K_{1} x_{1}(t)+x_{5}(t) x_{6}(t), \\
\mathcal{N}\left[x_{2}(t)\right] & =-K_{1} x_{2}(t)-x_{7}(t) x_{9}(t)-M x_{6}(t)+N x_{4}(t), \\
\mathcal{N}\left[x_{3}(t)\right] & =-K_{1} x_{3}(t)-x_{4}(t) x_{5}(t)+x_{7}(t) x_{8}(t), \\
\mathcal{N}\left[x_{4}(t)\right] & =-K_{1} x_{4}(t)-K x_{7}(t)+x_{2}(t) x_{6}(t)-x_{3}(t) x_{5}(t) \\
& +N x_{5}(t), \\
\mathcal{N}\left[x_{5}(t)\right] & =-K_{1} x_{5}(t)-x_{1}(t) x_{6}(t)+x_{3}(t) x_{4}(t)-N x_{4}(t), \\
\mathcal{N}\left[x_{6}(t)\right] & =-K_{1} x_{6}(t)+x_{1}(t) x_{5}(t)-x_{2}(t) x_{4}(t), \\
\mathcal{N}\left[x_{7}(t)\right] & =-K_{1} x_{7}(t)+x_{2}(t) x_{9}(t)-x_{3}(t) x_{8}(t), \\
\mathcal{N}\left[x_{8}(t)\right] & =-K_{1} x_{8}(t)-x_{1}(t) x_{9}(t)+x_{3}(t) x_{7}(t)-N x_{7}(t), \\
\mathcal{N}\left[x_{9}(t)\right] & =-K_{1} x_{9}(t)+x_{1}(t) x_{8}(t)-x_{2}(t) x_{7}(t),
\end{aligned}
$$

where $K>0$ and $K_{1}>0$ are unknown parameters, then the solutions of the Eq. (11) for the initial approximations $x_{i_{0}}, i=\overline{1,9}$ are given by:

$$
\begin{aligned}
x_{1_{0}}(t) & =\left[\frac{A_{1}}{M^{2}+N^{2}}\left(M^{2}+N^{2} \cos \left(\sqrt{M^{2}+N^{2}} t\right)\right)\right. \\
& -\frac{A_{2} N^{2}-2 A_{5}\left(M^{2}+N^{2}\right)}{N \sqrt{M^{2}+N^{2}}} \sin \left(\sqrt{M^{2}+N^{2}} t\right) \\
& -\frac{A_{3}}{2\left(M^{2}+N^{2}\right)}\left(-1+\cos \left(\sqrt{\left(M^{2}+N^{2}\right) t}\right)\right) \\
& +\frac{A_{5}}{N}\left(-M \sin (M t)+\sqrt{M^{2}+N^{2}} \sin \left(\sqrt{M^{2}+N^{2}} t\right)\right)
\end{aligned}
$$

$$
\begin{aligned}
& \left.-\frac{A_{6} M}{N}\left(\cos (M t)-\cos \left(\sqrt{M^{2}+N^{2}} t\right)\right)\right] \cdot e^{-K_{1} t}, \\
& x_{2_{0}}(t)=\left[\frac{A_{1} N}{\sqrt{M^{2}+N^{2}}} \sin \left(\sqrt{M^{2}+N^{2}} t\right)\right. \\
& +\left(A_{2}-\frac{2 A_{5}\left(M^{2}+N^{2}\right)}{N^{2}} \cos \left(\sqrt{M^{2}+N^{2}} t\right)\right. \\
& +\frac{A_{3} M}{2 \sqrt{M^{2}+N^{2}}} \sin \left(\sqrt{M^{2}+N^{2}} t\right) \\
& +\frac{A_{5}\left(M^{2}+N^{2}\right)}{N^{2}}\left(\cos (M t)+\cos \left(\sqrt{M^{2}+N^{2}} t\right)\right) \\
& +\frac{A_{6}}{N^{2}}\left(-\left(M^{2}+N^{2}\right) \sin (M t)\right. \\
& \left.\left.+M \sqrt{M^{2}+N^{2}} \sin \left(\sqrt{M^{2}+N^{2}} t\right)\right)\right] \cdot e^{-K_{1} t}, \\
& x_{3_{0}}(t)=\left[-\frac{A_{1} M N}{M^{2}+N^{2}}\left(-1+\cos \left(\sqrt{\left(M^{2}+N^{2}\right) t}\right)\right)\right. \\
& +\frac{A_{2} N^{2}-2 A_{5}\left(M^{2}+N^{2}\right)}{M N^{2} \sqrt{M^{2}+N^{2}}} \sin \left(\sqrt{M^{2}+N^{2}} t\right) \\
& +\frac{A_{3}}{2}\left(1+\cos \left(\sqrt{\left(M^{2}+N^{2}\right) t}\right)\right) \\
& -\frac{A_{5}}{N^{2}}\left(-M \sin (M t)+\sqrt{M^{2}+N^{2}} \sin \left(\sqrt{M^{2}+N^{2}} t\right)\right. \\
& \left.+\frac{A_{6} M^{2}}{N^{2}}\left(1-\cos \left(\sqrt{M^{2}+N^{2}} t\right)\right)\right] \cdot e^{-K_{1} t}, \\
& x_{4_{0}}(t)=\left[A_{4} \cos (K t)-A_{7} \sin (K t)\right. \\
& -\frac{K N A_{8}}{K^{2}-M^{2}}(\cos (K t)-\cos (M t)) \\
& \left.-\frac{N A_{9}}{K^{2}-M^{2}}(-M \sin (K t)+K \sin (M t))\right] \cdot e^{-K_{1} t}, \\
& x_{5_{0}}(t)=\left(A_{5} \cos (M t)-A_{6} \sin (M t)\right) \cdot e^{-K_{1} t}, \\
& x_{6_{0}}(t)=\left(A_{6} \cos (M t)+A_{5} \sin (M t)\right) \cdot e^{-K_{1} t} \text {, } \\
& x_{7_{0}}(t)=\left[A_{7} \cos (K t)+A_{4} \sin (K t)\right. \\
& +\frac{M N A_{9}}{-K^{2}+M^{2}}(\cos (K t)-\cos (M t)) \\
& \left.-\frac{N A_{8}}{K^{2}-M^{2}}(K \sin (K t)-M \sin (M t))\right] \cdot e^{-K_{1} t}, \\
& x_{8_{0}}(t)=\left(A_{8} \cos (M t)-A_{9} \sin (M t)\right) \cdot e^{-K_{1} t}, \\
& x_{9_{0}}(t)=\left(A_{9} \cos (M t)+A_{8} \sin (M t)\right) \cdot e^{-K_{1} t} \text {. }
\end{aligned}
$$

By substituting Eqs. (20) into Eqs. (19), we reach the following results:

$$
\begin{aligned}
\mathcal{N}\left[x_{1_{0}}(t)\right] & =-K_{1} x_{1_{0}}(t)+x_{5_{0}}(t) x_{6_{0}}(t), \\
\mathcal{N}\left[x_{2_{0}}(t)\right] & =-K_{1} x_{2_{0}}(t)-x_{7_{0}}(t) x_{9_{0}}(t) \\
& -M x_{6_{0}}(t)+N x_{4_{0}}(t), \\
\mathcal{N}\left[x_{3_{0}}(t)\right] & =-K_{1} x_{3_{0}}(t)-x_{4_{0}}(t) x_{5_{0}}(t)+x_{7_{0}}(t) x_{8_{0}}(t), \\
\mathcal{N}\left[x_{4_{0}}(t)\right] & =-K_{1} x_{4_{0}}(t)-K x_{7_{0}}(t)+x_{2_{0}}(t) x_{6_{0}}(t) \\
& -x_{3_{0}}(t) x_{5_{0}}(t)+N x_{5_{0}}(t), \\
\mathcal{N}\left[x_{5_{0}}(t)\right] & =-K_{1} x_{5_{0}}(t)-x_{1_{0}}(t) x_{60}(t) \\
& +x_{3_{0}}(t) x_{4_{0}}(t)-N x_{4_{0}}(t),
\end{aligned}
$$




$$
\begin{aligned}
\mathcal{N}\left[x_{6_{0}}(t)\right] & =-K_{1} x_{6_{0}}(t)+x_{1_{0}}(t) x_{5_{0}}(t)-x_{2_{0}}(t) x_{4_{0}}(t), \\
\mathcal{N}\left[x_{7_{0}}(t)\right] & =-K_{1} x_{7_{0}}(t)+x_{2_{0}}(t) x_{9_{0}}(t)-x_{3_{0}}(t) x_{8_{0}}(t), \\
\mathcal{N}\left[x_{8_{0}}(t)\right] & =-K_{1} x_{8_{0}}(t)-x_{1_{0}}(t) x_{9_{0}}(t) \\
& +x_{3_{0}}(t) x_{7_{0}}(t)-N x_{7_{0}}(t), \\
\mathcal{N}\left[x_{9_{0}}(t)\right] & =-K_{1} x_{9_{0}}(t)+x_{1_{0}}(t) x_{8_{0}}(t)-x_{2_{0}}(t) x_{7_{0}}(t) .
\end{aligned}
$$

Remark 4.1. It is easy to see that the nonlinear operators $\mathcal{N}\left[x_{i_{0}}(t)\right], i=\overline{1,9}$ can be written as linear combinations of the functions

$$
\begin{aligned}
& e^{-K_{1} t} \cdot \cos (M t), e^{-K_{1} t} \cdot \sin (M t), e^{-2 K_{1} t} \cdot \cos ^{2}(M t), \\
& e^{-2 K_{1} t} \cdot \sin ^{2}(M t), e^{-2 K_{1} t} \cdot \cos (M t) \sin (M t), \\
& e^{-K_{1} t} \cdot \cos (K t), e^{-K_{1} t} \cdot \sin (K t), e^{-2 K_{1} t} \cdot \cos (K t) \cos (M t), \\
& e^{-2 K_{1} t} \cdot \sin (K t) \sin (M t), e^{-2 K_{1} t} \cdot \cos (K t) \sin (M t), \\
& e^{-2 K_{1} t} \cdot \sin (K t) \cos (M t), e^{-K_{1} t} \cdot \cos \left(\sqrt{M^{2}+N^{2}} t\right), \\
& e^{-K_{1} t} \cdot \sin \left(\sqrt{M^{2}+N^{2}} t\right), e^{-2 K_{1} t} \cdot \cos ^{2}\left(\sqrt{M^{2}+N^{2}} t\right), \\
& e^{-2 K_{1} t} \cdot \sin ^{2}\left(\sqrt{M^{2}+N^{2}} t\right), \\
& e^{-2 K_{1} t} \cdot \cos \left(\sqrt{M^{2}+N^{2}} t\right) \cdot \sin \left(\sqrt{M^{2}+N^{2}} t\right), \\
& e^{-2 K_{1} t} \cdot \cos (M t) \cdot \sin \left(\sqrt{M^{2}+N^{2}} t\right), \\
& e^{-2 K_{1} t} \cdot \cos \left(\sqrt{M^{2}+N^{2}} t\right) \cdot \sin (M t), \\
& e^{-2 K_{1} t} \cdot \cos (K t) \cdot \sin \left(\sqrt{M^{2}+N^{2}} t\right), \\
& e^{-2 K_{1} t} \cdot \cos \left(\sqrt{M^{2}+N^{2}} t\right) \cdot \sin (K t)
\end{aligned}
$$

Although the Eq. (12) is a nonhomogeneous linear one, finding its solution could be a difficult problem.

In order to compute the functions $x_{i_{1}}\left(t, B_{s}, C_{s}\right), i=$ $\overline{1,9}$, by the Remark 4.1 and the previous Section, the first approximation $x_{i_{1}}, i=\overline{1,9}$ can be written as:

$$
\begin{aligned}
& x_{i_{1}}\left(t, B_{s}, C_{s}\right)= \\
& =\sum_{n=1}^{8}\left[B_{n} \cos (2 n+1) \omega t+C_{n} \sin (2 n+1) \omega t\right] \cdot e^{-K_{1} t}, \\
& i=\overline{1,9}, \quad s=\overline{1,8} .
\end{aligned}
$$

Now, using the Eqs. (20) and Eq. (22), the first-order analytic approximate solution given by Eq. (16) of the Eqs. (5) - (17) is well-determined and its becomes:

$$
\begin{aligned}
& \bar{x}_{i}\left(t, B_{s}, C_{s}\right)=x_{i_{0}}(t)+x_{i_{1}}\left(t, B_{s}, C_{s}\right), \\
& i=\overline{1,9}, \quad s=\overline{1,8} .
\end{aligned}
$$

\section{Numerical Examples and Discussions}

The goal of this section is to prove the accuracy and the validity of the OHAM technique. We make use of the com- parison of our approximate solutions with numerical results obtained via the fourth-order Runge-Kutta method in the following case, as follows: we consider the initial value problem given by the system (4) with initial conditions $A_{i}=0.001, i=\overline{1,9}, M=15$ and $N=20$; then, the convergence-control parameters $K, K_{1}, \omega, B_{i}, C_{i}$, $i=\overline{1,8}$ are optimally determined by means of the leastsquare method using the Wolfram Mathematica 9.0 software.

One can show that these approximate solutions given by Eq. (23) are weak $\epsilon$-approximate solutions by computing the numerical values of the integral of square residual function (to see the Table 4 from Appendix 6), i.e.

$$
\begin{aligned}
\int_{0}^{1} R_{i}^{2}(t) d t, & i=1, \ldots, 9, \text { where } \\
R_{1}(t) & =\dot{\bar{x}}_{1}(t)+\bar{x}_{5}(t) \bar{x}_{6}(t)+N \bar{x}_{2}(t)-N \bar{x}_{5}(t), \\
R_{2}(t) & =\overline{\bar{x}}_{2}(t)-\bar{x}_{7}(t) \bar{x}_{9}(t)+M \bar{x}_{3}(t)-N \bar{x}_{1}(t) \\
& -M \bar{x}_{6}(t)+N \bar{x}_{4}(t), \\
R_{3}(t) & =\overline{\bar{x}}_{3}(t)-\bar{x}_{4}(t) \bar{x}_{5}(t)+\bar{x}_{7}(t) \bar{x}_{8}(t) \\
& -M \bar{x}_{2}(t)+M \bar{x}_{5}(t), \\
R_{4}(t) & =\overline{\bar{x}}_{4}(t)+\bar{x}_{2}(t) \bar{x}_{6}(t)-\bar{x}_{3}(t) \bar{x}_{5}(t)+N \bar{x}_{5}(t), \\
R_{5}(t) & =\overline{\bar{x}}_{5}(t)-\bar{x}_{1}(t) \bar{x}_{6}(t)+\bar{x}_{3}(t) \bar{x}_{4}(t) \\
& -N \bar{x}_{4}(t)+M \bar{x}_{6}(t), \\
R_{6}(t) & =\overline{\bar{x}}_{6}(t)+\bar{x}_{1}(t) \bar{x}_{5}(t)-\bar{x}_{2}(t) \bar{x}_{4}(t)-M \bar{x}_{5}(t), \\
R_{7}(t) & =\overline{\bar{x}}_{7}(t)+\bar{x}_{2}(t) \bar{x}_{9}(t)-\bar{x}_{3}(t) \bar{x}_{8}(t)+N \bar{x}_{8}(t), \\
R_{8}(t) & =\dot{\bar{x}}_{8}(t)-\bar{x}_{1}(t) \bar{x}_{9}(t)+\bar{x}_{3}(t) \bar{x}_{7}(t) \\
& +M \bar{x}_{9}(t)-N \bar{x}_{7}(t), \\
R_{9}(t) & =\overline{\bar{x}}_{9}(t)+\bar{x}_{1}(t) \bar{x}_{8}(t)-\bar{x}_{2}(t) \bar{x}_{7}(t)-M \bar{x}_{8}(t),
\end{aligned}
$$

with $\bar{x}_{i}(t), i=1, \ldots 9$, given by Eq. (23).

- For $\bar{x}_{1}$ the convergence-control parameters are respectively:

$$
\begin{aligned}
B_{1} & =0.6870910041, B_{2}=-0.4489602178, \\
B_{3} & =-0.5460639209, B_{4}=0.1352692445, \\
B_{5} & =0.2275764517, B_{6}=-0.0091553612, \\
B_{7} & =-0.0517476470, B_{8}=0.0059904466, \\
C_{1} & =-0.6043513189, C_{2}=-0.6632485829, \\
C_{3} & =0.2759472577, C_{4}=0.3824870710, \\
C_{5} & =-0.0477875461, C_{6}=-0.1139833895, \\
C_{7} & =-0.0026759104, C_{8}=0.0144167229, \\
K & =2.4390311791, K_{1}=7.00700137 \cdot 10^{-6}, \\
\omega & =1.6655500501 .
\end{aligned}
$$


The first-order approximation of the solution given by the Eq. (16) becomes:

$$
\begin{aligned}
\bar{x}_{1}(t) & =e^{-K_{1} t}(0.6870910041 \cos (\omega t) \\
& -0.4489602178 \cos (3 \omega t) \\
& -0.5460639209 \cos (5 \omega t) \\
& +0.1352692445 \cos (7 \omega t) \\
& +0.2275764517 \cos (9 \omega t) \\
& -0.0091553612 \cos (11 \omega t) \\
& -0.0517476470 \cos (13 \omega t) \\
& +0.0059904466 \cos (15 \omega t)) \\
& +(-0.6043513189 \sin (\omega t) \\
& -0.6632485829 \sin (3 \omega t) \\
& +0.2759472577 \sin (5 \omega t) \\
& +0.3824870710 \sin (7 \omega t) \\
& -0.0477875461 \sin (9 \omega t) \\
& -0.1139833895 \sin (11 \omega t) \\
& -0.0026759104 \sin (13 \omega t) \\
& +0.0144167229 \sin (15 \omega t)) e^{-K_{1} t} \\
& +0.0017 e^{-K_{1} t} \sin (25 t) \\
& +0.00005 e^{-K_{1} t}(-15 \sin (15 t)+25 \sin (25 t)) \\
& -0.00075 e^{-K_{1} t}(\cos (15 t)-\cos (25 t)) \\
& -8 \cdot 10^{-7} e^{-K_{1} t}(-1+\cos (25 t)) \\
& +1.6 \cdot 10^{-6} e^{-K_{1} t}(225+400 \cos (25 t)) \\
& -0110
\end{aligned}
$$

\begin{tabular}{lccc}
\hline$t$ & $x_{3_{\text {numerical }}}$ & $\begin{array}{c}\bar{x}_{3_{\text {OHAM }}} \\
\text { given by Eq. (A.2) }\end{array}$ & $\epsilon_{\chi_{3}}$ \\
\hline 0 & 0.001 & 0.001 & 0 \\
$1 / 10$ & 0.0025974531 & 0.0026022393 & $4.7862 \cdot 10^{-6}$ \\
$1 / 5$ & 0.0002348415 & 0.0002348153 & $2.6224 \cdot 10^{-8}$ \\
$3 / 10$ & -0.0007638090 & -0.0007667133 & $2.9043 \cdot 10^{-6}$ \\
$2 / 5$ & 0.0065863087 & 0.0065892747 & $2.9660 \cdot 10^{-6}$ \\
$1 / 2$ & -0.0064337350 & -0.0064354063 & $1.6713 \cdot 10^{-6}$ \\
$3 / 5$ & 0.0072796814 & 0.0072795202 & $1.6129 \cdot 10^{-7}$ \\
$7 / 10$ & 0.0002519075 & 0.0002537876 & $1.8801 \cdot 10^{-6}$ \\
$4 / 5$ & -0.0054946676 & -0.0054968279 & $2.1603 \cdot 10^{-6}$ \\
$9 / 10$ & 0.0140979129 & 0.0140967988 & $1.1141 \cdot 10^{-6}$ \\
1 & -0.0136322814 & -0.0136370940 & $4.8125 \cdot 10^{-6}$ \\
\hline
\end{tabular}

Finally, Table 1 and Tables 2-3 (see Appendix 6), respectively, emphasizes the accuracy of the OHAM technique by comparing the approximate analytic solutions $\bar{x}_{3}, \bar{x}_{5}$ and $\bar{x}_{8}$ respectively presented above with the corresponding numerical integration values.

On the other hand, the approximate analytic solutions $\bar{x}_{2} \div \bar{x}_{9}$ can be found in Appendix 6 .

Remark 5.1. The Figures 1-9 present numerical comparisons between the analytical approximate solutions obtained by OHAM Method and numerical results obtained by Runge-Kutta 4-steps integrator. We can see that the analytical approximate solutions offer us the same numerical results as Runge-Kutta 4-steps integrator.
Table 2: The comparison between the approximate solutions $\bar{x}_{5}$ given by Eq. (A.4) and the corresponding numerical solutions for $M=15$ and $N=20$ (relative errors: $\epsilon_{\chi_{5}}=\left|\chi_{5_{\text {numerical }}}-\bar{\chi}_{5_{\text {OHAM }}}\right|$ )

\begin{tabular}{lccc}
\hline$t$ & $x_{5_{\text {numerical }}}$ & $\begin{array}{c}\bar{x}_{5_{\text {OHAM }}} \\
\text { given by Eq. }\end{array}$ & $\epsilon_{\chi_{5}}$ \\
\hline 0 & 0.001 & 0.001 & 0 \\
$1 / 10$ & -0.0006815591 & -0.0006815657 & $6.6566 \cdot 10^{-9}$ \\
$1 / 5$ & 0.0000919487 & 0.0000919509 & $2.1944 \cdot 10^{-9}$ \\
$3 / 10$ & 0.0005350379 & 0.0005350411 & $3.2094 \cdot 10^{-9}$ \\
$2 / 5$ & -0.0009504616 & -0.0009504672 & $5.6096 \cdot 10^{-9}$ \\
$1 / 2$ & 0.0009887716 & 0.0009887778 & $6.2250 \cdot 10^{-9}$ \\
$3 / 5$ & -0.0006335602 & -0.0006335632 & $2.9127 \cdot 10^{-9}$ \\
$7 / 10$ & 0.0000245359 & 0.0000245330 & $2.9506 \cdot 10^{-9}$ \\
$4 / 5$ & 0.0005972232 & 0.0005972309 & $7.6578 \cdot 10^{-9}$ \\
$9 / 10$ & -0.0009844000 & -0.0009843996 & $3.3406 \cdot 10^{-10}$ \\
1 & 0.0009815058 & 0.0009816680 & $1.6213 \cdot 10^{-7}$ \\
\hline
\end{tabular}

Table 1: The comparison between the approximate solutions $\bar{x}_{3}$ given by Eq. (A.2) and the corresponding numerical solutions for $M=15$ and $N=20$ (relative errors: $\left.\epsilon_{x_{3}}=\left|x_{3_{\text {numerical }}}-\bar{x}_{3_{\text {OHAM }}}\right|\right)$

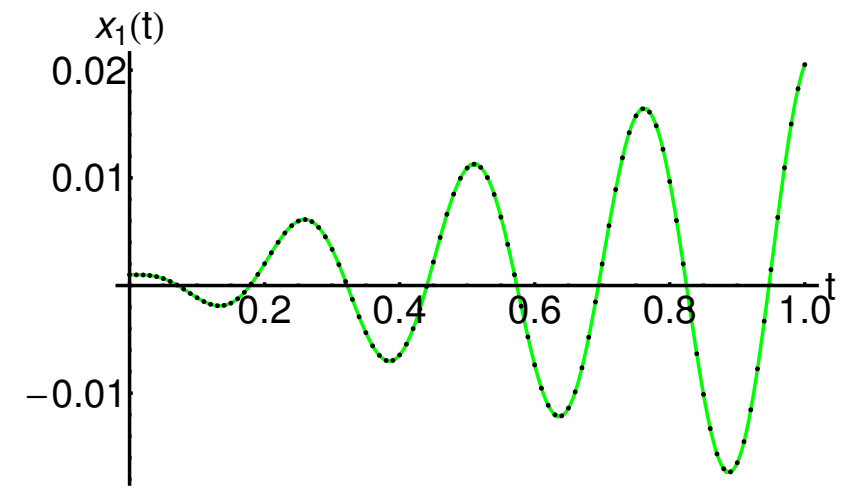

Figure 1: Comparison between the approximate solutions $\bar{x}_{1}$ given by Eq. (25) and the corresponding numerical solutions: numerical solution, ......... OHAM solution 
Table 3: The comparison between the approximate solutions $\bar{x}_{8}$ given by Eq. (A.7) and the corresponding numerical solutions for $M=15$ and $N=20$ (relative errors: $\left.\epsilon_{\chi_{8}}=\left|x_{8_{\text {numerical }}}-\bar{x}_{8_{\text {OHAM }}}\right|\right)$

\begin{tabular}{lccc}
\hline$t$ & $x_{8_{\text {numerical }}}$ & $\begin{array}{c}\bar{x}_{8_{\text {OHAM }}} \\
\text { given by Eq. (A.7) }\end{array}$ & $\epsilon_{x_{8}}$ \\
\hline $1 / 10$ & -0.0006815591 & -0.0006815657 & $6.6566 \cdot 10^{-9}$ \\
$1 / 5$ & 0.0000919487 & 0.0000919509 & $2.1944 \cdot 10^{-9}$ \\
$3 / 10$ & 0.0005350379 & 0.0005350411 & $3.2094 \cdot 10^{-9}$ \\
$2 / 5$ & -0.0009504616 & -0.0009504672 & $5.6096 \cdot 10^{-9}$ \\
$1 / 2$ & 0.0009887716 & 0.0009887778 & $6.2250 \cdot 10^{-9}$ \\
$3 / 5$ & -0.0006335602 & -0.0006335632 & $2.9127 \cdot 10^{-9}$ \\
$7 / 10$ & 0.0000245359 & 0.0000245330 & $2.9506 \cdot 10^{-9}$ \\
$4 / 5$ & 0.0005972232 & 0.0005972309 & $7.6578 \cdot 10^{-9}$ \\
$9 / 10$ & -0.0009844000 & -0.0009843996 & $3.3406 \cdot 10^{-10}$ \\
1 & 0.0009815058 & 0.0009816680 & $1.6213 \cdot 10^{-7}$ \\
\hline
\end{tabular}

Table 4: The numerical values of the integral of square residual function given by Eq. (24) corresponding to the approximate solutions given by Eqs. (25), (A.1)-(A.8) for $M=15$ and $N=20$.

\begin{tabular}{cc}
\hline$i$ & $\int_{0}^{1} R_{i}^{2}(t) d t$ \\
\hline 1 & $2.5681235091717695 \cdot 10^{-7}$ \\
2 & $6.4073772699416544 \cdot 10^{-6}$ \\
3 & $1.4346282316189278 \cdot 10^{-7}$ \\
4 & $1.083313166638645 \cdot 10^{-12}$ \\
5 & $2.4998964657011165 \cdot 10^{-12}$ \\
6 & $6.764954176305842 \cdot 10^{-13}$ \\
7 & $1.2075523363812237 \cdot 10^{-12}$ \\
8 & $2.5292599216354187 \cdot 10^{-12}$ \\
9 & $6.764881094330037 \cdot 10^{-13}$ \\
\hline
\end{tabular}

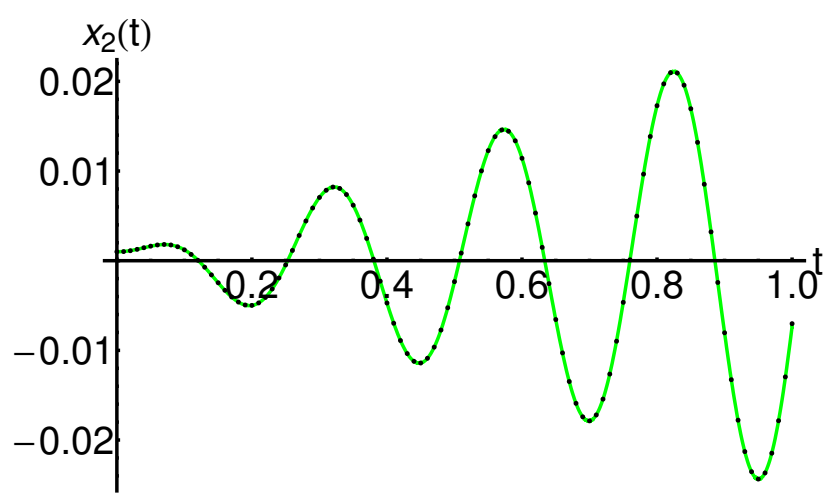

Figure 2: Comparison between the approximate solutions $\bar{x}_{2}$ given by Eq. (A.1) and the corresponding numerical solutions: numerical solution, $\cdots \ldots \ldots \ldots$ OHAM solution

\section{Conclusion}

In this paper, the stabilization of a dynamical system arising from an optimal control problem on the Lie group

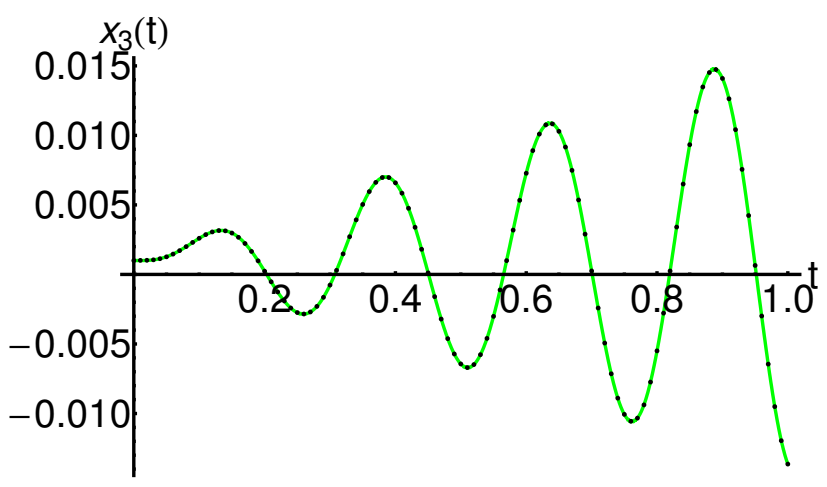

Figure 3: Comparison between the approximate solutions $\bar{x}_{3}$ given by Eq. (A.2) and the corresponding numerical solutions: numerical solution, ......... OHAM solution

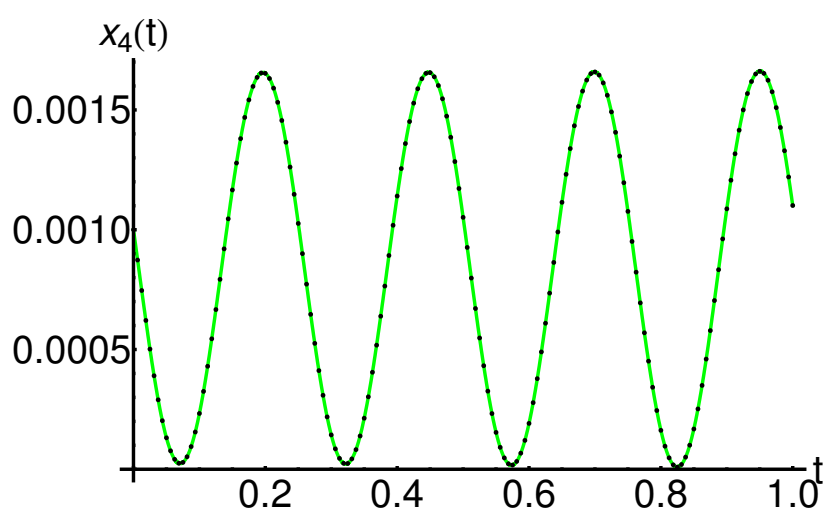

Figure 4: Comparison between the approximate solutions $\bar{x}_{4}$ given by Eq. (A.3) and the corresponding numerical solutions: - numerical solution,.$\ldots \ldots \ldots$ OHAM solution

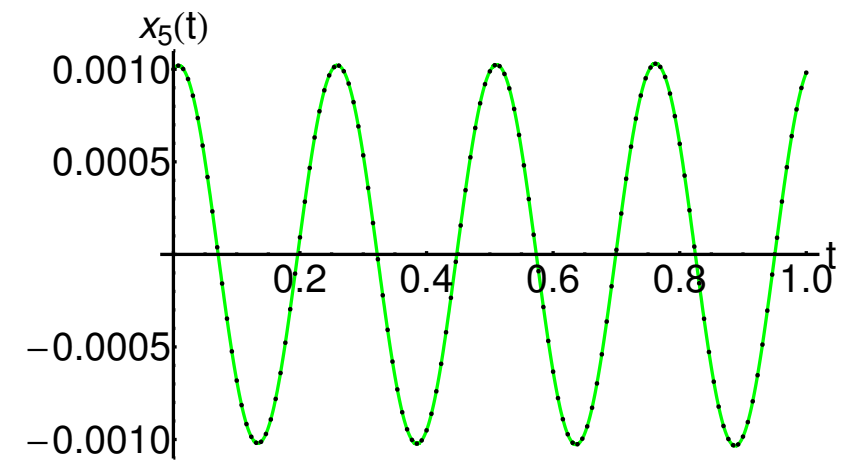

Figure 5: Comparison between the approximate solutions $\bar{x}_{5}$ given by Eq. (A.4) and the corresponding numerical solutions: numerical solution, $\cdots \cdots \cdots \cdots$ OHAM solution

$S O(3) \times \mathbb{R}^{3} \times \mathbb{R}^{3}$ is investigated. The conditions of the spectral and nonlinear stability of a specific equilibria were obtained using a linear control added to the initial system. Due to the existence of the Poisson formulation, the re- 
sults were obtained using specific tools, such as Arnold's method.

Finally, the analytic approximate solutions of the controlled dynamics are obtained via the Optimal Homotopy

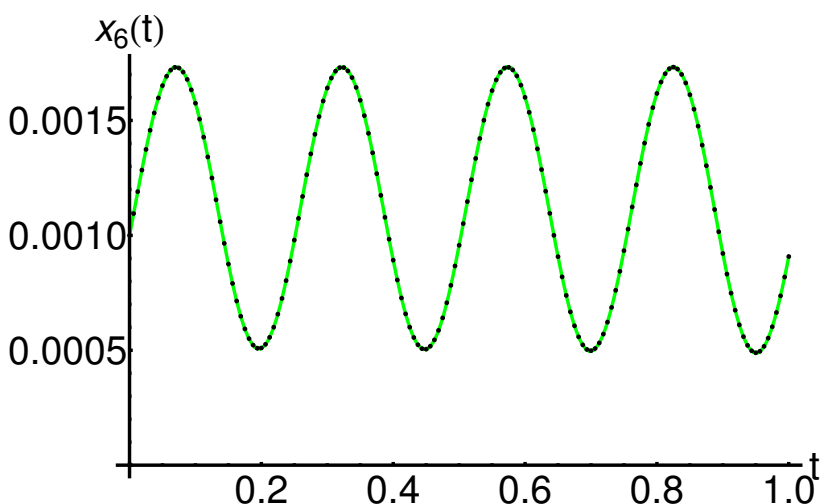

Figure 6: Comparison between the approximate solutions $\bar{x}_{6}$ given by Eq. (A.5) and the corresponding numerical solutions: numerical solution, ........ OHAM solution

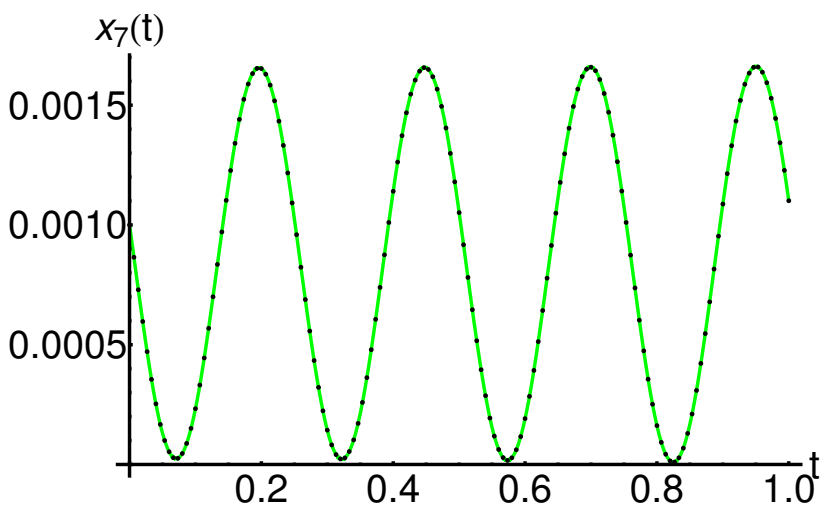

Figure 7: Comparison between the approximate solutions $\bar{x}_{7}$ given by Eq. (A.6) and the corresponding numerical solutions: numerical solution, ........ OHAM solution

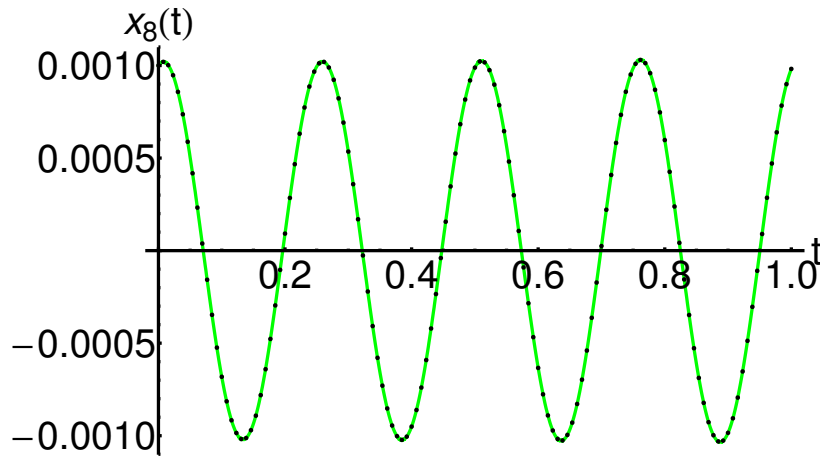

Figure 8: Comparison between the approximate solutions $\bar{\chi}_{8}$ given by Eq. (A.7) and the corresponding numerical solutions: numerical solution, ........ OHAM solution
Asymptotic Method. Numerical simulations and a comparison with Runge-Kutta 4-steps integrator are also presented.

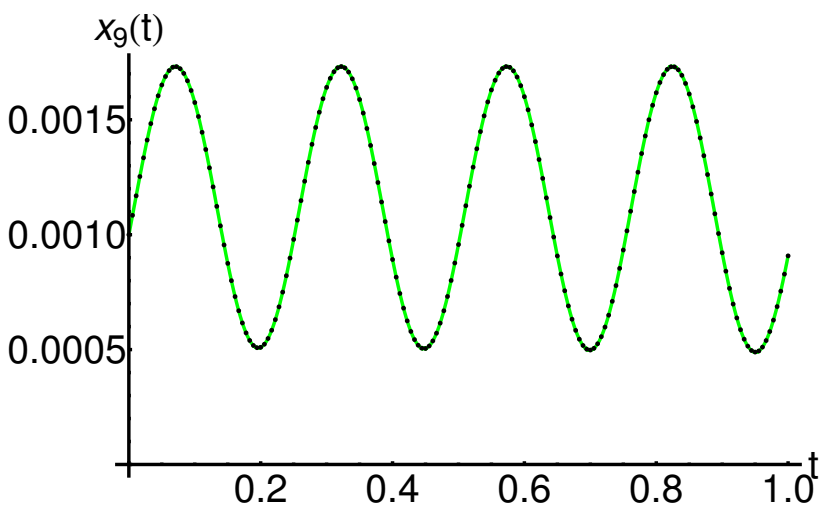

Figure 9: Comparison between the approximate solutions $\bar{\chi}_{9}$ given by Eq. (A.8) and the corresponding numerical solutions: numerical solution, ......... OHAM solution

Conflict of Interests: The authors declare that there is no conflict of interests regarding the publication of this paper.

\section{References}

[1] Biggs R., Remsing C. C., Control Systems on Three-Dimensional Lie Groups: Equivalence and Controllability, J. Dyn. Control Syst., 2014, 20(3), DOI 10.1007/s10883-014-9212-0.

[2] Biggs R., Remsing C. C., Some Remarks on the Oscillator Group, Differ. Geom. Appl., 2014, DOI 10.1016/j.difgeo.2014.03.003.

[3] Lăzureanu C., On a Hamiltonian version of controls dynamic for a drift-free left invariant control system on G4, Int. J. Geom. Methods M., 2012, 9(8), DOI 10.1142/S021988781250065X.

[4] Lăzureanu C., On a Hamilton-Poisson Approach of the MaxwellBloch Equations with a Control, Math. Phys. Anal. Geom., 2017, 20(3) DOI 10.1007/s11040-017-9251-3.

[5] Lăzureanu C., Hamilton-Poisson Realizations of the Integrable Deformations of the Rikitake System Adv. Math. Phys., 2017, 2017(10), 1-9.

[6] Pop C., Free Left Invariant Control System on the Lie Group $S O(3) \times \mathbb{R}^{3} \times \mathbb{R}^{3}$, Math. Probl. Eng., 2015, Volume 2015, Article ID 652819, doi:10.1155/2015/652819.

[7] Bellerose J., Scheeres D. J., Energy and stability in the full two body problem, Celest. Mech. Dyn. Astr., 2008, 100, 63-91.

[8] Birtea P., Hogea C., Puta M., Some Remarks on the Clebsch's System, Bull. Sci. Math., 2004, 128, 871-882.

[9] David D., Holm D., Multiple Lie-Poisson structures, reduction and geometric phases for the Maxwell-Bloch traveling wave equations, J. Nonlinear Sci., 1992, 2, 241-262.

[10] Puta M., On an Extension of the 3-Dimensional Toda Lattice, Preprint ESI, Vienna, 1996, 165, http://www.esi.ac.atstatic/ esiprpr/esi165.pdf. 
[11] Scheeres D. J., Stability of relative equilibria in the full two-body problem. Ann. N. Y. Acad. Sci., 2004, 1017, 81-94.

[12] Beck J. A., Hall C. D., Relative equilibria of a rigid satellite in a circular Keplerian orbit, J. Astronaut. Sci., 1998, 40(3), 215-247.

[13] Hirabayashi M., Morimoto M. Y., Yano H., et al., Linear stability of collinear equilibrium points around an asteroid as a two-connectedmass: application to fast rotating Asteroid 2000EB14. Icarus, 2010, 206, 780-782.

[14] Hughes P.C., Spacecraft Attitude Dynamics, John Wiley, New York, 1986, pp. 281-298.

[15] Leonard N. E., Averaging and motion control systems on Lie groups, Ph.D. Thesis, University of Maryland, College Park, MD, 1994.

[16] Dahleh M., Pierce A., Rabitz H. A., Ramakrishna V., Control of molecular motion, Proc. of the IEEE, 1996, 84(1), 7-15.

[17] Ghosh D., Banerjee S., Chowdhury A. R., Synchronization between variable time-delayed systems and cryptography, Europhys. Lett., 2007, 80(3), Article ID 30006, 6 pages.

[18] Ginoux J. M., Rossetto B., Differential geometry and mechanics: Applications to chaotic dynamical systems, Int. J. Bifurcat. Chaos., 2006, 16(4), 887-910.

[19] Ivan Ghe., Ivan M., Pop C., Numerical integration and Synchronization for the 3-Dimensional Metriplectic Volterra System, Math. Prob. Eng., 2011, vol. 2011, doi:10.1155/2011/723629.

[20] Pop Ariesanu C., The Synchronization of Two Metriplectic Systems Arisen from the Lü System, Advanced Engineering Forum Vols. 8-9 (2013), Trans. Tech. Publications, Switzerland, 2013, 471-479, doi:10.4028/www.scientific.net/AEF.8-9.471.

[21] Marsden J. E., Lectures on Mechanics, London Mathematical Society, Lecture Note Series, Cambridge University Press, Cambridge, 1992, vol. 174.

[22] Arnold V., Conditions for nonlinear stability of stationary plane curvilinear flows of an ideal fluid, Doklady, 1965, 162(5), 773777.

[23] Marinca V., Herisanu N., The Optimal Homotopy Asymptotic Method: Engineering Applications, Springer Verlag, Heidelberg, 2015.

[24] Marinca V., Herisanu N., Bota C., Marinca B., An optimal homotopy asymptotic method applied to the steady flow of a fourth grade fluid past a porous plate, Appl. Math. Lett., 2009, 22, 245-251.

[25] Elsgolts L., Differential Equations and the Calculus of Variations, Mir Publishers, Moscow, 1980.

[26] Bota C., Căruntu B., Approximate analytical solutions of the regularized long wave equation using the optimal homotopy perturbation method, Sci. World. J., 2014, Article ID: 721865, 6 pages.

\section{Appendix}

The following conditions $K_{1}=7.00700137 \cdot 10^{-6}$, $\omega=1.6655500501$ hold for all unknown functions $\bar{x}_{i}$, $i=\overline{1,9}$, so:

$$
\begin{aligned}
-f o r \bar{x}_{2}: & \\
\bar{x}_{2}(t) & =e^{-K_{1} t}(-0.4389432372 \cos (\omega t) \\
& +0.5020290567 \cos (3 \omega t) \\
& +0.2237312287 \cos (5 \omega t) \\
& -0.3256680044 \cos (7 \omega t) \\
& -0.0683191602 \cos (9 \omega t) \\
& +0.1147324797 \cos (11 \omega t) \\
& +0.0126641080 \cos (13 \omega t) \\
& -0.0202264714 \cos (15 \omega t)) \\
& -0.002125 e^{-K_{1} t} \cos (25 t) \\
& +0.0015625 e^{-K_{1} t}(\cos (15 t)+\cos (25 t)) \\
& +e^{-K_{1} t}(0.5027080265 \sin (\omega t) \\
& +0.3350256550 \sin (3 \omega t) \\
& -0.4352899954 \sin (5 \omega t) \\
& -0.1306954331 \sin (7 \omega t) \\
& +0.2103586786 \sin (9 \omega t) \\
& +0.0300134573 \sin (11 \omega t) \\
& -0.0576550229 \sin (13 \omega t) \\
& +0.0061020354 \sin (15 \omega t)) \\
& +0.0011 e^{-K_{1} t} \sin (25 t)+2.5 \\
& +10^{-6} e^{-K_{1} t}(-625 \sin (15 t)+375 \sin (25 t))
\end{aligned}
$$

- for $\bar{x}_{3}$ :

$$
\begin{aligned}
\bar{x}_{3}(t) & =e^{-K_{1} t}(-0.5160419993 \cos (\omega t) \\
& +0.3372418512 \cos (3 \omega t) \\
& +0.4100224611 \cos (5 \omega t) \\
& -0.1016916740 \cos (7 \omega t) \\
& -0.1702969101 \cos (9 \omega t) \\
& +0.0069229986 \cos (11 \omega t) \\
& +0.0388494598 \cos (13 \omega t) \\
& -0.0050061873 \cos (15 \omega t)) \\
& +(0.4538511906 \sin (\omega t) \\
& +0.4980536525 \sin (3 \omega t) \\
& -0.2073422773 \sin (5 \omega t) \\
& -0.2871797742 \sin (7 \omega t) \\
& +0.0364900541 \sin (9 \omega t) \\
& +0.0855759933 \sin (11 \omega t) \\
& +0.0019833945 \sin (13 \omega t) \\
& -0.0116955965 \sin (15 \omega t)) e^{-K_{1} t}
\end{aligned}
$$




$$
\begin{aligned}
& -0.001275 e^{-K_{1} t} \sin (25 t)-2.5 \\
& \cdot 10^{-6} e^{-K_{1} t}(-15 \sin (15 t)+25 \sin (25 t)) \\
& +0.0005625000 e^{-K_{1} t}(1-\cos (25 t)) \\
& -0.00048 e^{-K_{1} t}(-1+\cos (25 t)) \\
& +0.0005 e^{-K_{1} t}(1+\cos (25 t))
\end{aligned}
$$

- for $\bar{x}_{4}:$

$$
\begin{aligned}
\bar{x}_{4}(t) & =e^{-K_{1} t}(0.0014047411 \cos (\omega t) \\
& -0.0021067093 \cos (3 \omega t) \\
& -0.0001754437 \cos (5 \omega t) \\
& +0.0003714480 \cos (7 \omega t) \\
& +0.0004300377 \cos (9 \omega t) \\
& -0.0000814697 \cos (11 \omega t) \\
& -8.497125 \cdot 10^{-6} \cos (13 \omega t) \\
& +0.0001658929 \cos (15 \omega t)) \\
& +(0.0014137145 \sin (\omega t) \\
& +0.0002567584 \sin (3 \omega t) \\
& +0.0007210711 \sin (5 \omega t) \\
& +0.0001595064 \sin (7 \omega t) \\
& -0.0005212098 \sin (9 \omega t) \\
& -0.0000413490 \sin (11 \omega t) \\
& +0.0000357107 \sin (13 \omega t) \\
& -0.0008061102 \sin (15 \omega t)) e^{-K_{1} t} \\
& +0.001 e^{-K_{1} t} \cos (3.5665110571 t) \\
& +0.0003360195 e^{-K_{1} t}(\cos (3.5665110571 t) \\
& -\cos (15 t))-0.001 e^{-K_{1} t} \sin (3.5665110571 t) \\
& +0.0000942151 e^{-K_{1} t}(-15 \sin (3.5665110571 t) \\
& +3.5665110571 \sin (15 t)) \\
& .
\end{aligned}
$$$$
- \text { for } \bar{x}_{5} \text { : }
$$$$
\begin{aligned}
\bar{x}_{5}(t) & =e^{-K_{1} t}(0.0003950998 \cos (\omega t) \\
& -0.0001844516 \cos (3 \omega t) \\
& -0.0003597136 \cos (5 \omega t)+8.918677 \\
& \cdot 10^{-7} \cos (7 \omega t)-0.0008339975 \cos (9 \omega t) \\
& +0.0000152088 \cos (11 \omega t) \\
& -0.0000418091 \cos (13 \omega t) \\
& +0.0010087715 \cos (15 t)) \\
& +(-0.0003071207 \sin (\omega t)
\end{aligned}
$$$$
- \text { for } \bar{x}_{6}:
$$$$
\bar{x}_{6}(t)=e^{-K_{1} t}(0.0005541313 \cos (\omega t)
$$$$
+0.0006533041 \cos (3 \omega t)
$$$$
+0.0001646139 \cos (5 \omega t)
$$$$
-0.0002330474 \cos (7 \omega t)
$$$$
-0.0010805238 \cos (9 \omega t)
$$$$
+0.0000595280 \cos (11 \omega t)
$$$$
+6.366934 \cdot 10^{-6} \cos (13 \omega t)
$$$$
-0.0001243732 \cos (15 t))
$$$$
+(0.0014141404 \sin (\omega t)
$$$$
+0.0001011217 \sin (3 \omega t)
$$$$
-0.0004019421 \sin (5 \omega t)
$$$$
-0.0001317507 \sin (7 \omega t)
$$$$
-0.0008720894 \sin (9 \omega t)
$$$$
+0.0000307159 \sin (11 \omega t)
$$$$
-0.0000263820 \sin (13 \omega t)
$$$$
+0.0006045821 \sin (15 \omega t)) e^{-K_{1} t}
$$$$
+e^{-K_{1} t}(0.001 \cos (15 t)+0.001 \sin (15 t)) ;
$$$$
\text { - for } \bar{x}_{7}:
$$$$
\bar{x}_{7}(t)=e^{-K_{1} t}(-0.0007187903 \cos (\omega t)
$$$$
-0.0008984587 \cos (3 \omega t)
$$$$
-0.0002156138 \cos (5 \omega t)
$$$$
+0.0003149641 \cos (7 \omega t)
$$$$
+0.0014403433 \cos (9 \omega t)
$$$$
-0.0000798255 \cos (11 \omega t)
$$$$
-8.460837 \cdot 10^{-6} \cos (13 \omega t)
$$$$
+0.0001658418 \cos (15 \omega t))
$$$$
+(-0.0020538216 \sin (\omega t)
$$$$
-0.0001277630 \sin (3 \omega t)
$$ 
$+0.0005463646 \sin (5 \omega t)$

$+0.0001740129 \sin (7 \omega t)$

$+0.0011614636 \sin (9 \omega t)$

$-0.0000408001 \sin (11 \omega t)$

$+0.0000352719 \sin (13 \omega t)$

$-0.0008061122 \sin (15 \omega t)) e^{-K_{1} t}$

$+0.001 e^{-K_{1} t} \cos (0.1923247828 t)$

$+0.0013335525 e^{-K_{1} t}(\cos (0.1923247828 t)$

$-\cos (15 t))+0.001 e^{-K_{1} t} \sin (0.1923247828 t)$

$+0.0000889035 e^{-K_{1} t}(0.1923247828$

$\cdot \sin (0.1923247828 t)-15 \sin (15 t))$;

- for $\bar{x}_{8}$ :

$$
\begin{aligned}
\bar{x}_{8}(t) & =e^{-K_{1} t}(0.0003950998 \cos (1 \omega t) \\
& -0.0001844516 \cos (3 \omega t) \\
& -0.0003597136 \cos (5 \omega t) \\
& +8.918677 \cdot 10^{-7} \cos (7 \omega t) \\
& -0.0008339975 \cos (9 \omega t) \\
& +0.0000152088 \cos (11 \omega t) \\
& -0.0000418091 \cos (13 \omega t) \\
& +0.0010087715 \cos (15 \omega t)) \\
& +(-0.0003071207 \sin (\omega t) \\
& -0.0004137084 \sin (3 \omega t) \\
& +0.0000724864 \sin (5 \omega t) \\
& +0.0002604951 \sin (7 \omega t) \\
& +0.0010261920 \sin (9 \omega t) \\
& -0.0000865232 \sin (11 \omega t) \\
& -1.773634 \cdot 10^{-6} \sin (13 \omega t) \\
& +0.0002071073 \sin (15 \omega t)) e^{-K_{1} t} \\
& +0.001 e^{-K_{1} t} \cos (15 t)-0.001 e^{-K_{1} t} \sin (15 t) ;
\end{aligned}
$$

- for $\bar{x}_{9}$ :

$$
\begin{aligned}
\bar{x}_{9}(t) & =e^{-K_{1} t}(0.0005541313 \cos (\omega t) \\
& +0.0006533041 \cos (3 \omega t) \\
& +0.0001646139 \cos (5 \omega t) \\
& -0.0002330474 \cos (7 \omega t) \\
& -0.0010805238 \cos (9 \omega t) \\
& +0.0000595280 \cos (11 \omega t) \\
& +6.366933 \cdot 10^{-6} \cos (15 \omega t)
\end{aligned}
$$

$$
\begin{aligned}
& -0.0001243732 \cos (13 \omega t)) \\
& +(0.0014141404 \sin (1 \omega t) \\
& +0.0001011217 \sin (3 \omega t) \\
& -0.0004019421 \sin (5 \omega t) \\
& -0.0001317507 \sin (7 \omega t) \\
& -0.0008720894 \sin (9 \omega t) \\
& +0.0000307159 \sin (11 \omega t) \\
& -0.0000263820 \sin (13 \omega t) \\
& +0.0006045821 \sin (15 \omega t)) e^{-K_{1} t} \\
& +0.001 e^{-K_{1} t} \cos (15 t)+0.001 e^{-K_{1} t} \sin (15 t) ;
\end{aligned}
$$

